\title{
CALENDAR/MEETING NEWS
}

\author{
S/CAR 1994 \\ Winston-Salem, NC \\ June 12-15, 1994
}

For further information contact: Pat Rice, Department of Radiology, Bowman Gray School of Medicine, Medical Center Boulevard, Winston-Salem, NC 27157-1088. Telephone 919-716-2470.

\section{Editorial: The Role of Reviewers}

The reviewers listed below are pivotal in providing quality manuscripts to our readers. I extend to each and all of them my sincere thanks for their expert help in evaluating the submitted manuscripts. The volunteer efforts of each have been a very significant contribution.

\section{Reviewers for the Journal of Digital Imaging - 1992 through 1993}

Laurens Ackerman, M.D., Ph.D.

Robert M. Allman, M.D.

Ronald L. Arenson, M.D.

Gary Barnes, Ph.D.

David Volk Beard, Ph.D.

G. James Blaine, D.Sc.

Antonio Chiesa, M.D.

Glendon G. Cox, M.D.

Samuel J. Dwyer, III, Ph.D.

Peter Esser, Ph.D.

Elliot K. Fishman, M.D.

Jerry Froelich, M.D.

Gary D. Fullerton, Ph.D.

Maryellen L. Giger, Ph.D.

Thurman Gillespy III, M.D.

Robert E. Gold, M.D.

Reginald Greene, M.D.

Robert A. Greenes, M.D.

Peter Hahn, M.D., Ph.D.

Timothy J. Hall, Ph.D.

Marvin E. Haskin, M.D.

Atsuko Heshiki, M.D.

David G. Hill, Ph.D.

Karl-Heinz Hoehne, Ph.D.

Steven C. Horii, M.D.

H. K. Huang, D.Sc.

Tim B. Hunter, M.D.

Dragan Jezic, M.D.

R. Gilbert Jost, M.D.

Robert A. Kruger, Ph.D.
Heinz Lemke, Ph.D.

Gwilym S. Lodwick, M.D.

Tomoho Maeda, M.D.

Nicholas J. Mankovich, Ph.D.

Carroll R. Markivee, M.D.

David Martin, M.D.

Seong K. Mun, Ph.D.

Richard N. Nelson, M.D.

David Pickens, Ph.D.

William Palmer, M.D.

Deniese Parrish, M.P.H.

Henry P. Pendergrass, M.D.

John Perry, Ph.D.

Hans Roehrig, Ph.D.

Bruce Rosen, M.D., Ph.D.

Martin Sandler, M.D.

Roger H. Schneider, Ph.D.

Roger H. Shannon, M.D.

Donald K. Shaw, M.D.

Edward V. Staab, M.D.

A. Thomas Stavros, M.D.

Brent Stewart, Ph.D.

Jaime Taaffe, B.S.

Archibald Templeton, M.D.

Robert G. Thompson, B.S.

Jayaram K. Udupa, Ph.D.

Van Weeden, M.D., Ph.D.

Michael W. Vannier, M.D.

Robert F. Wagner, Ph.D. 